\title{
SOLVABILITY ON MANIFOLDS BY QUADRATURES PERMITTING ONLY INTEGRALS
}

\author{
BY KUO-TSAI CHEN ${ }^{1}$
}

Communiçated by S. Eilenberg, May 28, 1974

Let $M$ be a connected $C^{\infty}$ manifold, and let $\rho: \widetilde{M} \rightarrow M$ be the universal covering map. Choose a base point $\tilde{x}_{0} \in \widetilde{M}$, and write $x_{0}=$ $\rho \tilde{x}_{0}$. The fundamental group $\pi_{1}(M)$ is assumed to be finitely generated.

Let $A$ be a subcomplex of the de Rham complex $\Lambda(M)$ satisfying the conditions:

(a) The subcomplex $A$ is closed under the exterior product.

(b) The inclusion $A \subset \Lambda(M)$ induces an isomorphism $H(A) \approx H(\Lambda(M)$ ). Write $F_{0}=\rho^{*} A^{0}$ and $\Omega=\rho^{*} A^{1}$. If $w \in A^{1}$ is a closed 1-form on $M$, then the integral $\int_{\tilde{x}_{0}} \rho^{*} w$ is a function on $\widetilde{M}$ and can be regarded as a multivalued function on $M$. All such integrals together with 1 span a vector space $F_{1}$ of functions on $\widetilde{M}$ such that $F_{0} \subset F_{1}$. For $r \geqslant 1$, define $F_{r+1}$ to be the vector space of functions spanned by $F_{r}$ and all $\int_{\tilde{x}_{0}} w$, $w$ being closed 1 -forms belonging to the subspace $F_{r} \Omega$ of $\Lambda^{1}(\widetilde{M})$. It turns out that $\widetilde{F}=\bigcup_{r \geqslant 0} F_{r}$ is an algebra of functions on $\widetilde{M}$.

Recall that the lower central series of a group $G$ consists of commutator subgroups $G_{r}, r \geqslant 1$, defined by $G_{1}=G$ and $G_{r+1}=\left[G_{r}, G\right]$, $r \geqslant 1$. The lower central series is said to stabilize modulo torsion if $G_{r} / G_{r+1}$ is finite for $r$ sufficiently large. A group $G$ is said to be torsion free residually nilpotent if each quotient $G_{r} / G_{r+1}$ is torsion free and if $\bigcap_{G_{r}}=\{e\}$.

The purpose of this note is to announce the next results, which will be proved in detail elsewhere.

THEOREM 1. The algebra $\widetilde{F}$ is finitely generated over $F$ if and only

AMS (MOS) subject classifications (1970). Primary 58C99; Secondary 53C65, 55 A10, 58A10.

Key words and phrases. Differential forms, iterated integration, residually nilpotent groups, universal covering space, Picard-Vessiot theory.

1 Work supported in part by the National Science Foundation under NSF GP-34257. 
if the lower central series of $\pi_{1}(M)$ stabilizes modulo torsion.

THEOREM 2. The algebra $\widetilde{F}$ of functions on $\widetilde{M}$ separates $\widetilde{M}$ if and only if $\pi_{1}(M)$ is torsion free residually nilpotent.

COROllary 1. The algebra $\widetilde{F}$ is finitely generated and separates $\widetilde{M}$ if and only if $\pi_{1}(M)$ is torsion free nilpotent.

COROLlaRY 2. If $M$ is a compact Riemann surface, then $\widetilde{F}$ separates the universal covering surface $\widetilde{M}$.

The function algebra $\widetilde{F}$ is obtained from the given function algebra $F$ on $M$ by adjoining multivalued functions which are obtained through iterated integration. According to the above theorems, we know precisely when $\widetilde{F}$ can be obtained from $F$ by adjoining a finite number of elements and also when every continuous function on $\widetilde{M}$ can be approximated on compact sets by functions in $\widetilde{F}$. Thus our results provide answers to questions pertaining to a several independent variable version of the Picard-Vessiot theory. In the one variable case, it is known [6] that an extension of a differential field by integrals corresponds to a Galois group which is algebraic nilpotent.

Since a compact nilmanifold has a torsion free nilpotent fundamental group, this work also relates to the function theory on nilpotent Lie groups under a discrete subgroup action such as the continuous theta function theory by Auslander and Rauch [1].

In order to prove Theorems 1 and 2 , observe that $\widetilde{F}$ can be regarded as an algebra of iterated integrals of 1-forms on $M$, whose value along each path depends only on the path homotopy class. By restricting to the space of loops at $x_{0}$, we obtain from $\widetilde{F}$ a quotient algebra $\widetilde{F}^{\prime}$ which has an ascending filtration. We may take $\widetilde{F}^{\prime}$ as an algebra of functions on $\pi_{1}(M)$.

Theorem 1 is equivalent to a necessary and sufficient condition for $\widetilde{F}^{\prime}$ being finitely generated over the real (or complex) number field, and Theorem 2 reduces to a necessary and sufficient condition for $\widetilde{F}^{\prime}$ to separate $\pi_{1}(M)$. It remains to show that $\widetilde{F}^{\prime}=F_{A}^{\prime}$, where $F_{A}^{\prime}$ is defined as in [3]. The inclusion $\widetilde{F}^{\prime} \subset F_{A}^{\prime}$ is not difficult to see. Using a method of formal power series connections as described in [5], we are able to establish $F_{A}^{\prime} \subset$ $\widetilde{F}^{\prime}$.

Corollary 2 follows from Theorem 2 because of a result of Baumslag [2] which implies that $\pi_{1}(M)$ is torsion free residually nilpotent. 


\section{BIBLIOGRAPHY}

1. L. Auslander and H. E. Rauch, Theta functions or how to study functions on nilmanifolds and not even know it (mimeographed notes).

2. G. Baumslag, On the residual finiteness of generalised free products of nilpotent groups, Trans. Amer. Math. Soc. 106 (1963), 193-209. MR 26 \#2489.

3. K. T. Chen, Fundamental groups, nilmanifolds and iterated integrals, Bull. Amer. Math. Soc. 79 (1973), 1033-1035. MR 47 \#676.

4. - Iterated integrals, fundamental groups and covering spaces (to appear).

5. - Connections, holonomy and path space homology, Proc Sympos. Pure Math., vol. 27, Amer. Math. Soc., Providence, R. I., 1974.

6. E. R. Kolchin, Algebraic matric groups and the Picard-Vessiot theory of homogeneous linear ordinary differential equations, Ann. of Math. (2) 49 (1948), 1-42. MR 9, 561.

7. - Differential algebra and algebraic groups, Academic Press, New York, 1973.

DEPARTMENT OF MATHEMATICS, UNIVERSITY OF ILLINOIS, URBANA, ILLINOIS 61801 